\title{
Chondrocutaneous posterior auricular artery perforator free flap for single-stage reconstruction of the nasal tip: a case report
}

Jun Yong Lee ${ }^{1,2}$,

Jeong Hwa Seo ${ }^{1,3}$,

Sung-No Jung ${ }^{1,3}$,

Bommie Florence Seo ${ }^{1,3}$

${ }^{1}$ Department of Plastic and

Reconstructive Surgery, College of

Medicine, The Catholic University of

Korea, Seoul; ${ }^{2}$ Department of Plastic

and Reconstructive Surgery, Incheon St.

Mary's Hospital, Incheon; ${ }^{3}$ Department

of Plastic and Reconstructive Surgery,

Uijeongbu St. Mary's Hospital,

Uijeongbu, Korea

\begin{abstract}
Full-thickness nasal tip reconstruction is a challenging process that requires provision of ample skin and soft tissue, and intricate cartilage structure that maintains its architecture in the long term. In this report, we describe reconstruction of a full-thickness nasal tip and ala defect using a posterior auricular artery perforator based chondrocutaneous free flap. The flap consisted of two lay ers of skin covering conchal cartilage, and was based on a perforating branch of the posterior auricular artery. A superficial vein was secured at the posterior margin. The donor perforator was anastomosed to a perforating branch of the lateral nasal artery. The superficial vein was connected to a superficial vein of the surrounding soft tissue. The donor healed well after primary closure. The flap survived without complications, and the contour of the nasal rim was sustained at followup 6 months later. As opposed to combined composite reconstructions using a free cartilage graft together with a small free flap or pedicled nasolabial flap, the posterior auricular artery perforator free flap encompasses all required tissue types, and is similar in contour to the alar area. This flap is a useful option in single-stage reconstruction of nasal composite defects.
\end{abstract}

Keywords: Case reports / Free tissue flaps / Nose deformities, acquired / Reconstructive surgical procedures

\section{INTRODUCTION}

Reconstruction of full-thickness defects of the nasal tip is very challenging because of its intricate cartilage structure, ample but thin soft tissue lining, and contoured nasal rim. This three dimensional architecture composed of aesthetic subunits is difficult to recreate [1].

A variety of options have been utilized for full-thickness nasal tip reconstruction. Pedicled flaps such as the classical forehead flap and nasolabial flap, composite grafts, pedicled flaps and

\footnotetext{
Correspondence: Bommie Florence Seo

Department of Plastic and Reconstructive Surgery, Uijeongbu St. Mary's Hospital, College of Medicine, The Catholic University of Korea, 271 Cheonbo-ro, Uijeongbu 11765 , Korea

E-mail: bommiefseo@catholic.ac.kr

Received November 11, 2021 / Revised November 21, 2021 / Accepted December 18, 2021
}

small free flaps combined with free cartilage grafts and skin grafts have been described [2,3]. However, pedicled flaps leave behind scars in the facial area, which are more prominent in the Asian population, and combined flaps with cartilage free grafts do not guarantee sufficient vascularity to the cartilage. Composite auricular chondrocutaneous grafts, while useful for coverage of defects smaller than $2 \mathrm{~cm}$ in size, are inadequate for larger defects [4].

The helix has been introduced as a donor for nose reconstruction in the past as both are chondrocutaneous structures. The majority of helical free flaps subsequently described in the literature are centered on the ascending helical rim, based on the superficial temporal vessels. Because the pedicle is short and thin, surgeons have attempted to overcome this limitation by using retrograde flow designs, additional proximal dissection of 
the pedicle, or utilizing supermicrosurgery $[5,6]$.

As the auricle also receives supply from the posterior auricular artery, the authors designed a new flap centered on the conchal region of the ear, with the aim of providing a three-layered, single-stage reconstructive option for the patient in our case.

\section{CASE REPORT}

A 25-year-old man presented with a nasal tip amputation caused by a human bite, without the amputated part (Fig. 1). The patient lived overseas, and to shorten the duration of treatment, we planned reconstruction using a single-stage fullthickness chondrocutaneous free flap based on a posterior auricular artery perforator. Reconstruction was performed after irrigation and debridement, while administering intravenous antibiotics (amoxicillin clavulanate). Dimensions of the halfmoon shaped defect were $2.5 \times 2.5 \times 0.5 \mathrm{~cm}$, extending from the left nasal tip to the ala. The depleted nasal lining was $0.5 \times 1 \mathrm{~cm}$. After tracing of the posterior auricular artery and its branches with a handheld Doppler, the flap was designed so that conchal skin would become the nasal lining, conchal cartilage would substitute the alar cartilage and posterior auricular skin would reconstruct the external skin. The recipient vessel was a branch of the lateral nasal artery with comparable diameter, which was
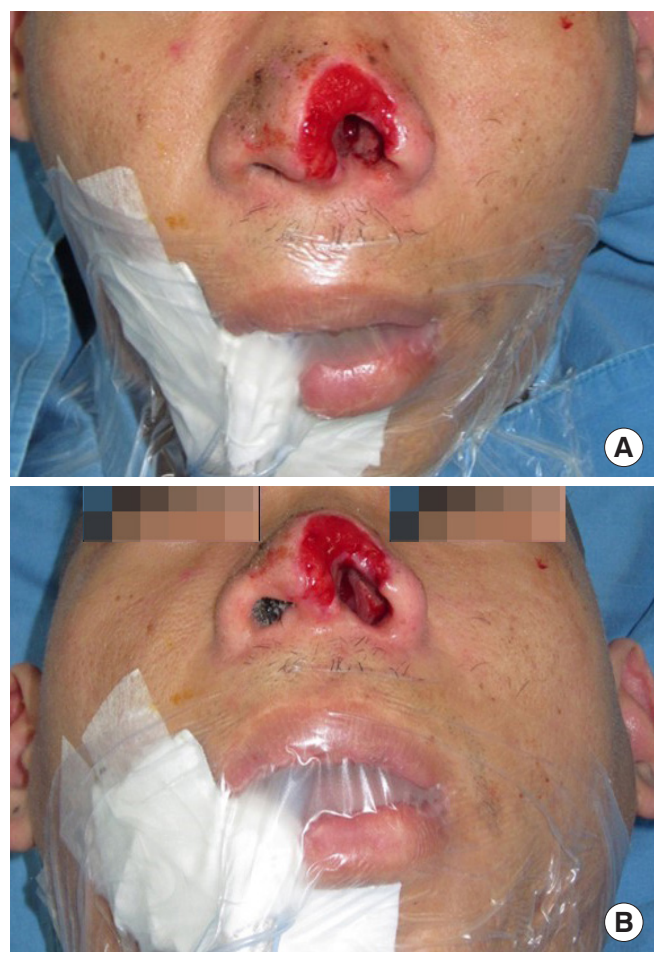

Fig. 1. A 25-year-old man with a full-thickness nasal tip defect, extending from the left nasal tip to the mid-ala, including the soft triangle. (A) Frontal view. (B) Worm's eye view. anastomosed to the posterior auricular artery perforator in end-to-end manner, using 10-0 Ethilon sutures. A subcutaneous vein was anastomosed to a subcutaneous vein surrounding the defect (Fig. 2). The donor site underwent primary closure. Systemic heparinization was maintained for 3 days postoperatively. The flap and donor healed well without complications (Figs. 3, 4).

\section{DISCUSSION}

Full-thickness defects of the nasal tip require reconstruction of three layers: the skin, the cartilaginous framework, and the mucosal lining. Pedicled flaps, such as the paramedian forehead
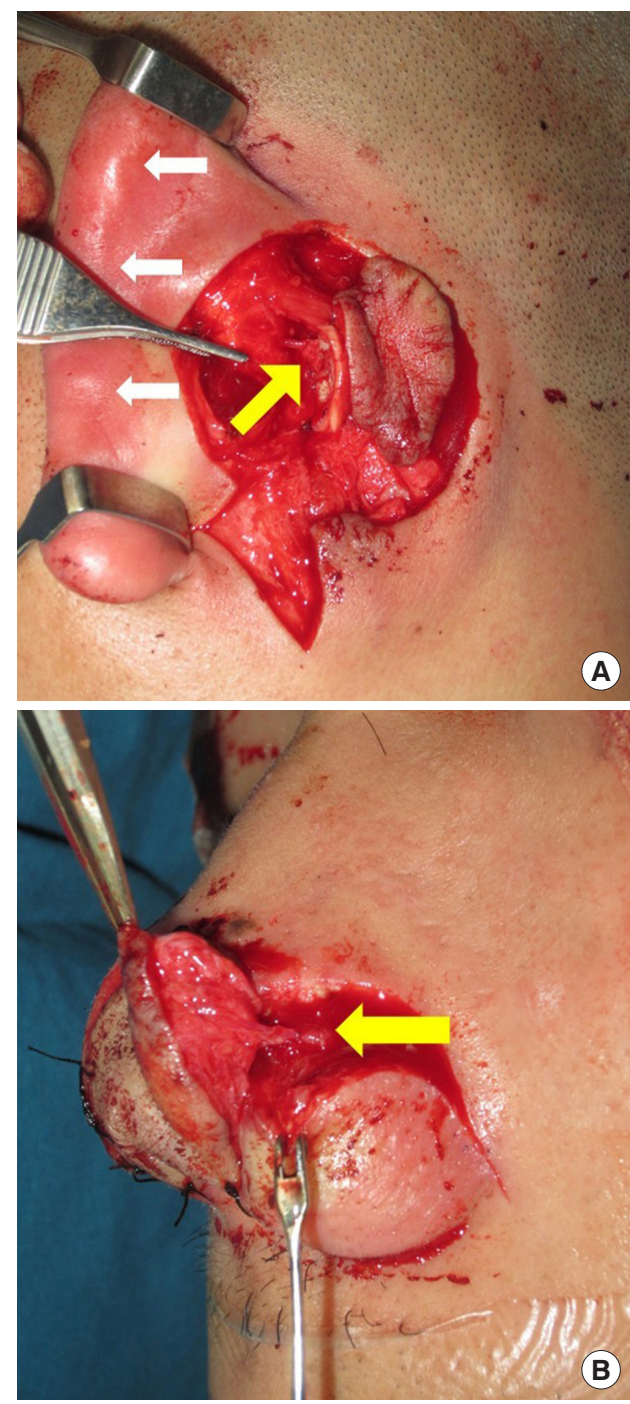

Fig. 2. Intraoperative photographs. (A) The posterior surface of the left auricle is seen, with the helix (white arrows) pulled forward with retractors. The postauricular flap is based on the posterior auricular artery perforator (yellow arrow). (B) After anastomosis between the posterior auricular perforator and a branch of the lateral nasal artery (yellow arrow). 


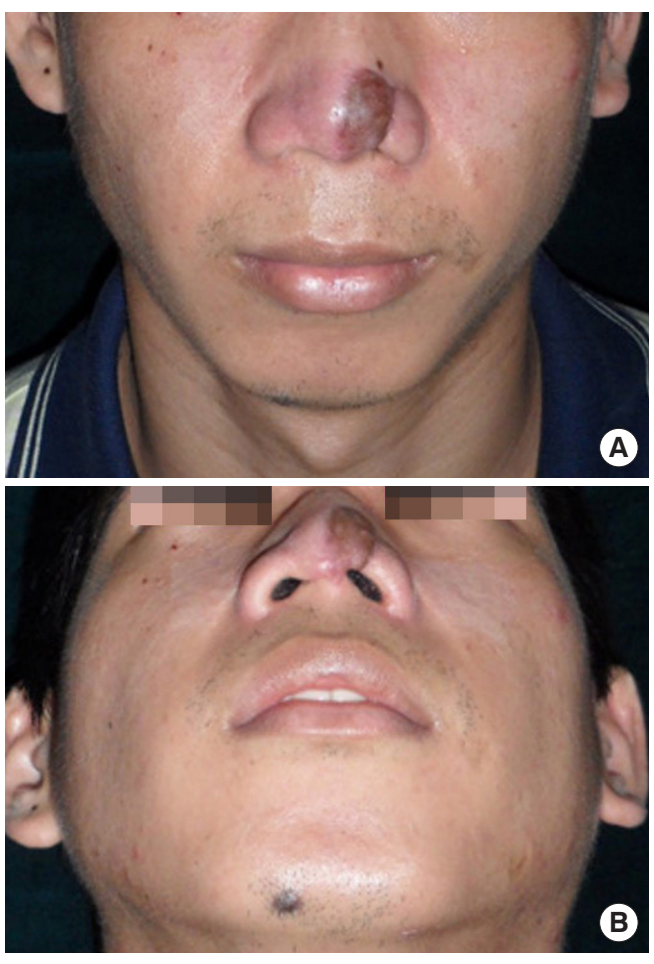

Fig. 3. Follow-up photographs at 10 weeks showing hyperpigmentation of the flap but near normal contour. (A) Frontal view. (B) Worm's eye view.

flap, and the nasolabial flap are safe options, but are bulky, create a prominent scar in the facial area, and do not provide framework tissue or nasal lining. Folding the tip of the pedicled flap may allow formation of a double lining, but may compromise the flap, especially when located in the distal tip [7].

Chondrocutaneous composite grafts of the helix, may be a satisfactory alternative. This method is easy to perform, provides structural framework as well as soft tissue, and has been excellent solutions for smaller defects [8]. However, lack of stable vascularity precludes the use of this method in larger defects.

A variety of small free flaps have been mentioned as microvascular reconstruction options for nasal defects. The first dorsal metacarpal flap, dorsalis pedis flap, radial or ulnar artery perforator flaps are all thin flaps that may be folded to provide double lining $[9,10]$. However, these free flaps all require incorporation of bone or cartilage grafts. Most of the time, reconstruction is a staged procedure, beginning with incorporation of the free flap into the defect, creating external and internal lining. The structural framework is inserted either during the free flap procedure or afterwards, when the flap has stabilized. Ancillary operations regarding refining nasal contour can follow these steps.

The auricular chondrocutaneous free flap, first introduced by
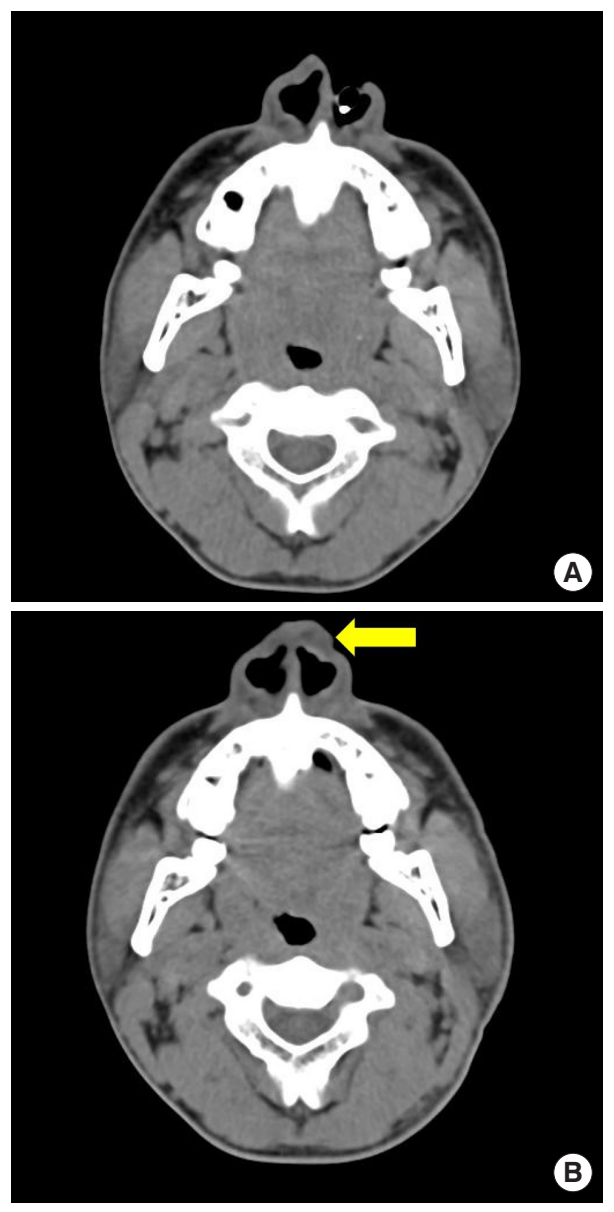

Fig. 4. Computed tomography. (A) Preoperative image. (B) Postoperative image showing the replenished nasal tip with good contour (yellow arrow).

Lin et al. [11] using the accessory auricle as a free flap, was subsequently adopted as a viable option for single-stage, three-layer reconstruction of the nose. Most reports describe the flap as centered on the anterior root of the helix, elevating the superficial temporal artery and vein as its pedicle. The proximal vessels will be incorporated for anterograde flow, and the distal vessels may be harvested for retrograde flow, based on the recipient site and inset [8]. Issues related to this flap are the prominence of the donor scar at the front of the ear, which at times require $s$ a secondary flap from the auricular region for coverage. Another obstacle is the relatively short length of the pedicle, and the search for a sufficiently sized recipient vessel in the vicinity of the defect.

As an alternative, the posterior auricular artery chondrocutaneous flap that was used in this case enabled the surgeons to obtain three-layered tissue from the conchal region, which is much more advantageous in obscuring the donor scar. The branch and perforator of the posterior auricular artery was harvested, and anastomosed in perforator-to-perforator fashion, 
therefore depleting the need for a long pedicle.

Limitations of this method are that microsurgical expertise is essential to achieve successful results, and that even with good flap vascularization and healing, shrinkage of flap volume has been reported [6]. In our case, the patient presented with pigmentation of the external skin, even though he had not experienced wound problems during the postoperative period. This may be due to perfusion irregularities in the process of flap stabilization, or the patient's skin type, and may be temporary, but owing to the departure of the patient, we were unable to obtain long-term results.

As opposed to combined composite reconstructions using a free cartilage graft together with a small free flap or pedicled nasolabial flap, the posterior auricular artery perforator free flap encompasses all required tissue types, and is similar in contour to the alar area. Unlike superficial temporal artery based helical free flaps, the donor site can be hidden in the concavity of the concha and behind the helix. This flap is a useful option in single-stage reconstruction of nasal composite defects.

\section{NOTES}

\section{Conflict of interest}

Jun Yong Lee and Sung-No Jung are editorial board member of the journal but were not involved in the peer reviewer selection, evaluation, or decision process of this article. No other potential conflicts of interest relevant to this article were reported.

\section{Ethical approval}

The study was approved by the Institutional Review Board of Catholic Medical Center (IRB No. UC21ZASA0131).

\section{Patient consent}

The patient provided written informed consent for the publication and the use of his images.

\section{ORCID}

Jun Yong Lee

Jeong Hwa Seo

https://orcid.org/0000-0002-6967-4447

Sung-No Jung

https://orcid.org/0000-0002-0419-4717
Bommie Florence Seo https://orcid.org/0000-0002-6907-5962

\section{Author contribution}

Conceptualization: Bommie Florence Seo. Data curation: Jun Yong Lee. Formal analysis: Jun Yong Lee, Jeong Hwa Seo. Visualization: Jeong Hwa Seo. Writing - original draft: Jun Yong Lee. Writing - review \& editing: Sung-No Jung, Bommie Florence Seo. Supervision: Sung-No Jung, Bommie Florence Seo.

\section{REFERENCES}

1. Kim TK, Jeong JY. Surgical anatomy for Asian rhinoplasty. Arch Craniofac Surg 2019;20:147-57.

2. Senturk E, Dagistanli N, Calim OF, Ozturan O. Nasal reconstruction following a dog bite. J Craniofac Surg 2019;30:22335.

3. Lim J, Oh J, Eun S. Flap reconstruction of soft tissue defect after resecting a huge hemangioma of the nose. Arch Craniofac Surg 2020;21:69-72.

4. Driscoll BP, Baker SR. Reconstruction of nasal alar defects. Arch Facial Plast Surg 2001;3:91-9.

5. Castello JR, Taglialatela Scafati S, Sanchez O. Bilateral nasal ala reconstruction of the cocaine-injured nose with 2 free reverseflow helical rim flaps. Ann Plast Surg 2014;73:304-6.

6. Jeong HH, Choi DH, Hong JP, Suh HS. Use of a helical composite free flap for alar defect reconstruction with a supermicrosurgical technique. Arch Plast Surg 2018;45:466-9.

7. Menick FJ. A 10-year experience in nasal reconstruction with the three-stage forehead flap. Plast Reconstr Surg 2002;109: 1839-55.

8. Zhang YX, Yang J, Wang D, Ong YS, Follmar KE, Zhang Y, et al. Extended applications of vascularized preauricular and helical rim flaps in reconstruction of nasal defects. Plast Reconstr Surg 2008;121:1589-97.

9. Beahm EK, Walton RL, Burget GC. Free first dorsal metacarpal artery flap for nasal lining. Microsurgery 2005;25:551-5.

10. McCraw JB, Furlow LT Jr. The dorsalis pedis arterialized flap: a clinical study. Plast Reconstr Surg 1975;55:177-85.

11. Lin SD, Lin GT, Lai CS, Hsu PJ. Nasal alar reconstruction with free “accessory auricle”. Plast Reconstr Surg 1984;73:827-9. 